\title{
Which Electrospray-Based Ionization Method Best Reflects Protein-Ligand Interactions Found in Solution? A Comparison of ESI, nanoESI, and ESSI for the Determination of Dissociation Constants with Mass Spectrometry
}

\author{
Matthias Conradin Jecklin, David Touboul, Cédric Bovet, \\ Arno Wortmann, and Renato Zenobi \\ Department of Chemistry and Applied Biosciences, ETH Zurich, Zurich, Switzerland
}

\begin{abstract}
We present a comparison of three different electrospray-based ionization techniques for the investigation of noncovalent complexes with mass spectrometry. The features and characteristics of standard electrospray ionization (ESI), chip-based nanoESI, and electrosonic spray ionization (ESSI) mounted onto a hybrid quadrupole time-of-flight mass spectrometer were compared in their performance to determine the dissociation constant $\left(K_{\mathrm{D}}\right)$ of the model system hen egg white lysozyme (HEWL) binding to $N, N^{\prime}, N^{\prime \prime}$-triacetylchitotriose $\left(\mathrm{NAG}_{3}\right)$. The best $K_{\mathrm{D}}$ value compared with solution data were found for ESSI, $19.4 \pm 3.6 \mu \mathrm{M}$. Then, we determined the $K_{\mathrm{D}} \mathrm{S}$ of the two nucleotide binding sites of adenylate kinase (AK), where we obtained $K_{\mathrm{D}} \mathrm{S}$ of $2.2 \pm 0.8 \mu \mathrm{M}$ for the first and $19.5 \pm 8.0 \mu \mathrm{M}$ for the second binding site using ESSI. We found a weak charge state dependence of the $K_{\mathrm{D}}$ for both protein-ligand systems, where for all ionization techniques the $K_{\mathrm{D}}$ value decreases with increasing charge state. We demonstrate that ESSI is very gentle and insensitive to instrumental parameters, and the $K_{\mathrm{D}}$ obtained is in good agreement with solution phase results from the literature. In addition, we tried to determine the $K_{\mathrm{D}}$ for the lymphocyte-specific kinase LCK binding to a kinase inhibitor using nanoESI due to the very low amount of sample available. In this case, we found $K_{\mathrm{D}}$ values with a strong charge state dependence, which were in no case close to literature values for solution phase. (J Am Soc Mass Spectrom 2008, 19, 332-343) (c) 2008 American Society for Mass Spectrometry
\end{abstract}

$\mathrm{T}$ he discovery of electrospray ionization (ESI) [1] and its ability to transfer high molecular weight biomolecules into the gas-phase revolutionized biological mass spectrometry (MS) and is still a constantly growing area of research. Earlier ESI sources dispersed liquids into aerosols by forcing a dissolved sample liquid through a hypodermic needle using a syringe pump (at typical flow rate of 5 to $20 \mu \mathrm{L} / \mathrm{min}$ ) and by applying a spray voltage (usually 3 to $4 \mathrm{kV}$ ) on the spray capillary tip $[1,2]$. Such electrospray purely driven by the applied potential can form different spray modes, such as dripping, stable cone-jet and multi-jet modes [3]. Vertes' group showed how spray voltages and modes can be tuned to achieve different ion yields and spray stabilities and how they affect the spectrum quality [4, 5]. Modern ESI interfaces, however, bypass these phenomena to some extent as they are pneumat-

Address reprint requests to Dr. Renato Zenobi, Department of Chemistry and Applied Biosciences, ETH, Hönggerberg, HCI, CH-8093 Zurich, Switzerland. E-mail: zenobi@org.chem.ethz.ch ically assisted by a concentric flow of an inert gas (usually nitrogen) around the electrospray plume. The use of a "nebulizer" or "sheath" gas was first proposed by Bruins et al. [6].

Miniaturization of the electrospray source, the development of nanoelectrospray ionization (nanoESI), as first described by Wilm and Mann, and coworkers [7-9], brought considerable advantages to study biomolecules with MS. The main difference compared with conventional ESI is the use of very small spray capillaries (1 to $5 \mu \mathrm{m}$ i.d.), that work with very low sample flow rates (20 to $100 \mathrm{~nL} / \mathrm{min}$ ), leading to a drastic reduction in sample consumption. The liquid sample flow is sometimes driven solely by capillary forces or slightly supported by a gentle backing pressure on the capillary, but no coaxial sheath gas is needed. However, the comparatively time-consuming procedure of manual capillary loading is still a limitation for high sample throughput. A chip-based nanoelectrospray (nanoES) source (NanoMate; Advion Bioscience, Ithaca, NY), in contrast, allows fast and automatic loading of the sam- 
ple by combining a spray chip—a micromachined silicon wafer-with a pipeting robot [10]. This technology, with its ability of LC-coupling, offers great advantages for automation and high-throughput protein characterization and proteomics [11]. Conventional ESI produces initial droplets of 1 to $5 \mu \mathrm{m}$ diameter [12], while nanoESI generates initial droplets with a diameter less than $200 \mathrm{~nm}$, which are about 100 to 1000 times smaller in volume [8]. A higher number of droplets generated in nanoES will form more ions that are available for MS since smaller initial droplets accelerate the desolvation process. The higher ionization efficiency is also due to the higher concentration of excess charge on the droplets [13].

In 2004, Takats [14] introduced a set-up for electrosonic spray ionization (ESSI), which is a hybrid of sonic spray ionization [15, 16] and ESI. It employs a traditional micro ESI source with supersonic nebulizer gas surrounding the spray tip. This technique creates ultrafine initial droplets with a very efficient subsequent desolvation of the droplets to form ions. Takats and coworkers reported very narrow charge state distributions, considerably less sensitivity to salt clustering effects, and very narrow peak widths for the examined model proteins [14]. They could even provide evidence that fully desolvated ions were generated at atmospheric pressure with ESSI. ESSI has been reported to transfer intact biomolecular complexes from solution to the gas phase [17].

ESI-MS is also known to transfer noncovalent complexes from solution into the gas phase [18-24]. However, whether the conformation of these macromolecules and their noncovalent interactions reflects the actual binding conditions in solution is still a highly debated question [25-32].

A lot of effort is currently put into developing approaches to quantitatively measure noncovalent interaction strengths of protein/peptide-ligand systems by ESI-MS [33-37]. Improvements for accurate determination of dissociation constants $\left(K_{D}\right)$ s have been presented, e.g., taking into account different ionization efficiencies of free protein and noncovalent complexes $[38,39]$, or correcting for nonspecific ligand binding $[40,41]$.

In this work, for verification of our methodology, we first applied the titration method [33] to study the noncovalent interaction in a well-characterized protein ligand system, hen egg white lysozyme (HEWL) binding to $N, N^{\prime}, N^{\prime \prime}$-triacetylchitotriose $\left(\mathrm{NAG}_{3}\right)$. HEWL catalyses the hydrolysis of $\beta$-1,4-glycosidic linkages in certain gram-positive bacterial walls and is a bacteriological agent in hen egg white $[42,43]$. Three different ionization methods, ESI, nanoESI, and ESSI were investigated in terms of their influence on complex stability in the gas phase and to see which one best reflects the protein complex equilibrium found in solution. In most work found in the literature, $K_{\mathrm{D}} \mathrm{s}$ are determined from the ratio of complex and free protein, using either a single (dominant) charge state or mass-deconvoluted spectra. It is also interesting to study whether the calculated $K_{\mathrm{D}}$ varies with individual charge state. In a well-behaved system, little or no charge state dependence should exist. However, differences in ionization efficiency, in transmission efficiency of the mass spectrometer for different species, and charge-dependent modulation of interaction with a ligand in the gas phase may exist in some cases. In this work, we therefore calculated $K_{\mathrm{D}}$ f for individual charge states. The HEWL$\mathrm{NAG}_{3}$ interactions was found to be well-behaved in this respect.

We extended this study to determine the binding affinity of adenylate kinase (AK) binding to adenosine$5^{\prime}$-diphosphate (ADP). AK is a phosphotransferase involved in cellular energy homeostasis and is regulating the production of ADP from adenosine- 5 '-triphosphate under the presence of adenosine- 5 ' monophosphate and has two binding sites for these nucleotides [44]. We compared the three ionization techniques and again calculated the $K_{\mathrm{D}} \mathrm{S}$ in a charge state-resolved manner. We observed only a small increase in affinity with increasing charge on the protein; the lowest $K_{\mathrm{D}}$ values were found for ESSI.

In addition we tried to apply the ESI-MS titration method to quantify the binding strength of a kinase inhibitor ("Compound A", whose name and structure cannot be given because of corporate issues) to the SRC-family lymphocyte-specific kinase LCK. Because of the considerably higher sample consumption of ESI and ESSI, this investigation was restricted to nanoESI. LCK is involved in the initial phosphorylation of T-cell receptor components, which is required for signal transduction and T-cell activation. Hence, it is an attractive cell-specific target for the design of T-cell immunosuppressants $[45,46]$. Using the same method, we calculated $K_{\mathrm{D}}$ values for the apparent charge states of LCK and Compound A. This case was found to be problematic: the calculated $K_{\mathrm{D}}$ values showed a strong charge state dependence.

\section{Experimental}

\section{Instrumentation}

Mass spectrometric analysis was performed with a hybrid quadrupole time-of-flight mass spectrometer (Q-TOF Ultima; Waters/Micromass Ltd., Manchester, UK) equipped with a Z-spray interface. The instrument was controlled via the MassLynx version 4.0 software. All measurements were performed in the positive ion mode. The source temperature was kept at $50{ }^{\circ} \mathrm{C}$ to $55^{\circ} \mathrm{C}$ for ESSI and nanoESI measurements. For ESI measurements, a higher source temperature of $90^{\circ} \mathrm{C}$ was used for a complete desolvation of the protein ions. The cone voltage was kept at $45 \mathrm{~V}$ for all the measurements, since no influence on the complex stability or desolvation process was observed. The RF1 voltage, the potential applied to the first ion tunnel in the linear flight path before the quadrupole, the collision cell and 
the TOF-tube, was varied from 20 to $200 \mathrm{~V}$. Argon was used as collision gas and a typical setting of $20 \mathrm{~V}$ was used for the collision energy parameter for optimized desolvation. The transmission of the ions was optimized on the quadrupole for the required mass range ( $m / z 1500$ to 3500 for HEWL, m/z 2000 to 4500 for AK). Mass spectra were accumulated during 2 to $3 \mathrm{~min}$ to have good signal-to-noise ratio. Calibration of the instrument up to $6000 \mathrm{~m} / \mathrm{z}$ was performed using the cesium iodide (CsI) clusters generated by spraying a solution of CsI in water/2-propanol (1/1, vol/vol) at a concentration of $2 \mu \mathrm{g} / \mu \mathrm{L}$.

ESI measurements were performed using the manufacturer's standard ESI source (Waters/Micromass Ltd.). The i.d. of the spray capillary was $75 \mu \mathrm{m}$ and the spray distance was around $1 \mathrm{~cm}$.

The ESSI source was, with some modifications, rebuilt as described by Takats [14]. The voltage supply was achieved by a stainless steel union (Upchurch Scientific Inc., Oak Harbor, WA) instead of applying it directly to the tip of the syringe. Teflon was chosen as ferrule material (instead of graphite) due to its high flexibility and softness for an optimal sealing of the high pressures in the T-Element. The T-Element was purchased from Swagelok (Solon, $\mathrm{OH}$ ). Untreated fusedsilica tubing with standard polyimide coating (350 $\mu \mathrm{m}$ o.d. and $250 \mu \mathrm{m}$ i.d. for the outer capillary; $150 \mu \mathrm{m}$ o.d. and $50 \mu \mathrm{m}$ i.d. for the sample capillary) and ferrules were obtained from BGB Analytik (Böckten, Switzerland). The ESSI source was mounted on a $x-y-z$ positioning stage and was carefully aligned to the MS inlet to achieve the highest sensitivity. A spray distance of 8 to $10 \mathrm{~cm}$ was used for all ESSI measurements. Nitrogen was used for the sonic nebulizing gas at a typical pressure of 15 to 20 bar, which led to a gas flow rate of $\sim 2.5$ to $3.5 \mathrm{~L} / \mathrm{min}$ on the spray tip.

A syringe pump (Harvard 22 syringe pump; Harvard Apparatus GmbH, March-Hugstetten, Germany) was used for sample infusion using ESI and ESSI.

NanoESI measurements were performed using an automated chip-based nanoESI robot (NanoMate model 100; Advion Bioscience, Ithaca, NY). It holds a 96-well sample plate, a rack of 96 disposable, conductive pipette tips, and a nanospray chip containing $20 \times 20$ nozzles of $5 \mu \mathrm{m}$ diameter. A gentle backing pressure of 4 to 6 bar on the spray tip was used to assist the liquid sample flow.

\section{Materials and Methods}

Hen egg white lysozyme (HEWL; $\mathrm{MW} \approx 14.3 \mathrm{kDa}$ ), adenylate kinase from chicken muscle $(\mathrm{AK} ; \mathrm{MW} \approx 24.4$ $\mathrm{kDa}$ ), adenosine-5'-diphosphate (ADP; $\mathrm{MW}=427.2$ $\mathrm{Da})$, disodium salt hydrate, $N, N^{\prime}, N^{\prime \prime}$-triacetylchitotriose (NAG3; $\mathrm{MW}=627.6 \mathrm{Da}$ ), as well as CsI and ammonium bicarbonate were purchased form Sigma-Aldrich Chemie $\mathrm{GmbH}$, Buchs, Switzerland). LCK (MW $\approx 30.6 \mathrm{kDa}$ ) and Compound A were provided by the Novartis Institutes for Biomedical Research.
All mass spectra for the titration experiments were recorded under nondenaturing conditions using a 20 $\mathrm{mM}$ ammonium bicarbonate buffer at $\mathrm{pH} 6.8$ ( $\mathrm{pH}$ was adjusted using dry ice). Before mass spectrometric analysis, HEWL, AK, and LCK samples were desalted using Micro Bio-Spin 6 columns (BioRad, Hercules, CA) with a $6 \mathrm{kDa}$ cutoff equilibrated with the $20 \mathrm{mM}$ ammonium bicarbonate buffer at $\mathrm{pH} 6.8$.

Titration experiments were performed keeping the protein concentration constant $(10 \mu \mathrm{M}$ for both HEWL and $\mathrm{AK}$ ) and varying the ligand concentration (from 1 to $50 \mu \mathrm{M}$ ADP for AK and 1 to $100 \mu \mathrm{M}$ NAG3 for HEWL). Mass spectra were first background subtracted and smoothed using the MassLynx software. Second, peak areas for free protein and the noncovalent complexes were integrated using JDPeakIntegrator, a software developed by in our laboratory especially for this purpose [34].

The model for the $K_{\mathrm{D}}$ calculation has been adapted from Daniel et al. [34]. The ratio of the free protein signal over complex signal was plotted against the concentration of ligand and titration curves obtained that way were fitted using the following equation:

$$
\begin{aligned}
& \frac{I(P)}{I(\mathrm{PL})}= \\
& -\frac{-K_{D}+[K]_{0}-[P]_{0}-\sqrt{4 K_{D}[L]_{0}+\left(K_{D}-L_{0}+[P]_{0}\right)^{2}}}{2 L_{0}}
\end{aligned}
$$

where $\mathrm{I}(\mathrm{P}) / \mathrm{I}(\mathrm{PL})$ stands for the intensity ratio of free protein over complex, $[\mathrm{P}]_{0}$ indicates the initial protein concentration, $[\mathrm{L}]_{0}$ the initial ligand concentration and $K_{\mathrm{D}}$ was the fitting parameter. We obtained different titration points by plotting the ratio of free protein over complex as a function of added ligand. By fitting these titration curves using eq 1 , we calculated the $K_{\mathrm{D}}$ values for the HEWL-NAG3 system and the binding of ADP to the first binding site of $\mathrm{AK}$. To determine the $K_{\mathrm{D}}$ value of ADP for the second binding site of AK, we took into account the already bound ADP in the first binding pocket. Therefore, we subtracted $10 \mu \mathrm{M}$ off the initial ADP concentration, as the first binding pocket of the protein $\left(\right.$ as $\left.[\mathrm{P}]_{0}=10 \mu \mathrm{M}\right)$ was fully saturated.

It was assumed for all cases that the ionization efficiency is the same for complex and free protein. The mass of the ligand is very small compared with the proteins mass and therefore the ligand will not interfere greatly with the ionization processes of the protein surface. Furthermore, this assumption allowed us to use the intensity ratios of free protein over complex instead of their concentrations in solution; no response factor had to be introduced. The $K_{\mathrm{D}}$ calculation and titration curve fitting were performed using Origin v7.5 software (OriginLab Corp., Northampton, MA). 


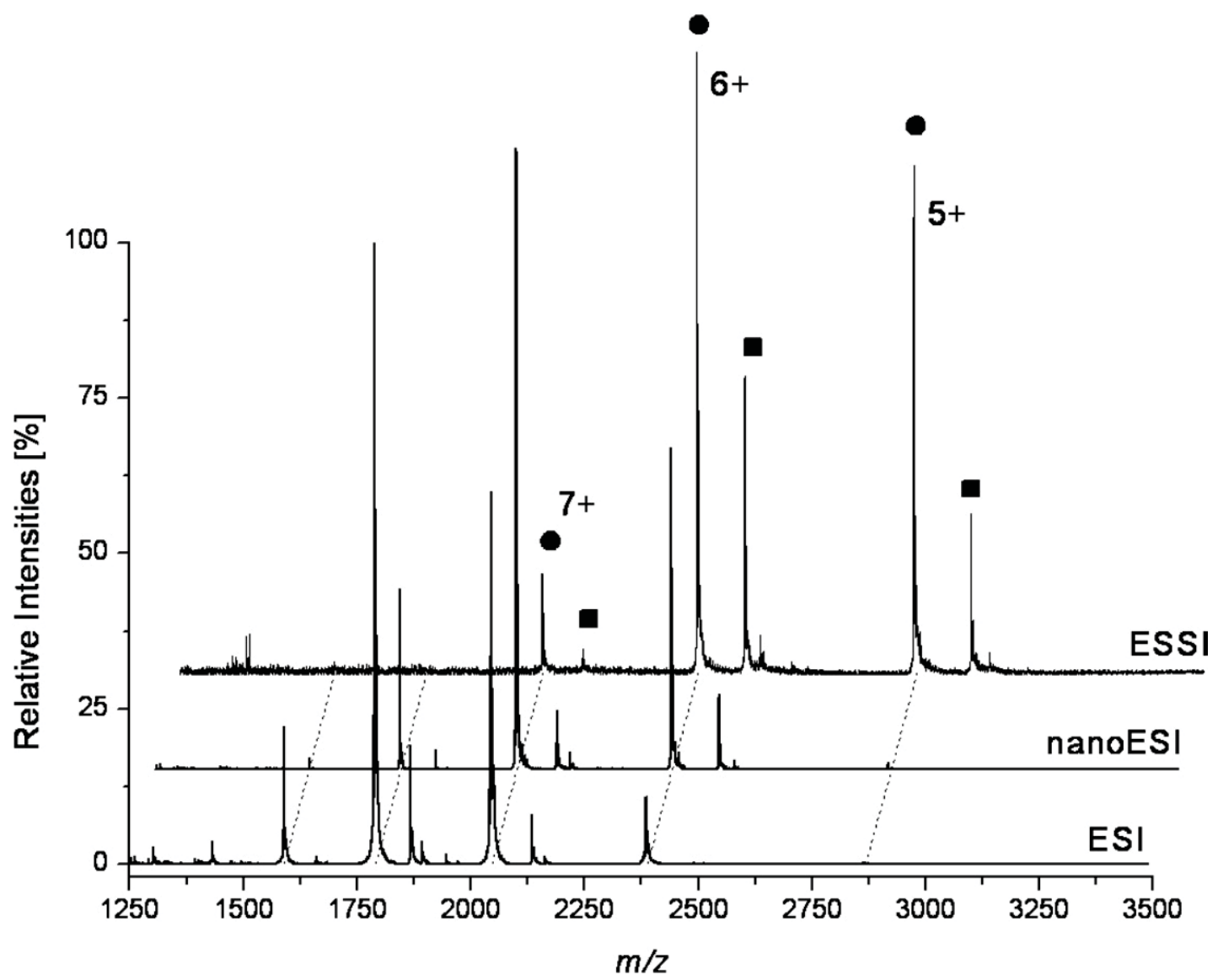

Figure 1. Representative mass spectra of the noncovalent HEWL-NAG 3 complex (HEWL $10 \mu \mathrm{M}$, $\mathrm{NAG}_{3} 60 \mu \mathrm{M}$ in $20 \mathrm{mM}$ ammonium bicarbonate buffer) using different ionization techniques. The charge states of free protein signals (filled circle) and $\mathrm{HEWL}-\mathrm{NAG}_{3}$ complex signals (filled square]) are given for ESSI.

\section{Results and Discussion}

\section{Binding of $H E W L$ with $N A G_{3}$}

Representative mass spectra of the noncovalent complexes between HEWL and $\mathrm{NAG}_{3}$ are shown in Figure 1. The most abundant charge state is shifted comparing ESI, nanoESI, and ESSI. The most intense charge state for ESI is +8 , appearing together with $+9,+7$, and +6 . For nanoESI the most intense signal is obtained for +7 , which is observed together with +9 (very low) $,+8,+6$, and +5 (very low). For ESSI the predominant protein signal corresponds to charge state +6 , which appears together with +7 and +5 . By comparing the same charge state $(+6)$ for the different ionization techniques in Figure 1, a variation in complex abundance is noted.
The highest signal for the HEWL-NAG ${ }_{3}$ complex is observed for ESSI, where the signal for the complex is $>50 \%$ compared with the free protein signal. For ESI and nanoESI the complex signal never exceeds approximately $20 \%$ of the corresponding free protein signal. This is evidence for the softness of ESSI. Due to the fast desolvation process of ESSI, noncovalent interactions present in solution can be "frozen" faster and transferred into the gas phase more rapidly. Noncovalent interaction would thus be less disturbed and the complexes will be better conserved after transfer into the gas phase.

The $K_{\mathrm{D}}$ values determined for the titration of HEWL with $\mathrm{NAG}_{3}$ are given in Table 1 . The errors indicate the fitting quality according to eq 1 (see the Experimental

Table 1. Calculated dissociation constants $\left(\mathrm{K}_{\mathrm{D}} \mathrm{s}\right)$ in $\mu \mathrm{M}$ from the titration experiments of HEWL with $\mathrm{NAG}_{3}$ using different ionization techniques and constant instrumental parameters (RF1 voltage $100 \mathrm{~V}$ ). The $\mathrm{K}_{\mathrm{D}}$ values were determined for the individual charges states of the protein. The error values given represent the fitting quality

\begin{tabular}{lcccrr}
\hline Charge state & +8 & +7 & +6 & +5 & Mean $_{\mathrm{D}}$ \\
\hline \hline ESSI & & & $18.1 \pm 1.3$ & $20.6 \pm 0.5$ & $19.4 \pm 3.6$ \\
NanoESI & $38.3 \pm 2.1$ & $41.1 \pm 1.8$ & $40.0 \pm 5$ & $39.8 \pm 8.8$ \\
ESI & $46.6 \pm 2.8$ & $72.9 \pm 6.0$ & & $59.8 \pm 17.6$
\end{tabular}


section). The $K_{\mathrm{D}}$ values agree quite well with the values of HEWL binding $\mathrm{NAG}_{3}$ in solution. According to the literature, $K_{\mathrm{D}}$ values have been obtained using different methods: $6 \mu \mathrm{M}$ using diffusion MS [47], $6.6 \mu \mathrm{M}$ using UV [48], and $8.6 \mu \mathrm{M}$ using fluorescence [49]. Dennhart and Letzel demonstrated a $K_{\mathrm{D}}$ determination for the same protein ligand system by ESI titration MS and reported values in the range of 12 to $27 \mu \mathrm{M}$ using different evaluation methods and different incubation times [43]. The values reported in this work, especially the ones obtained for the ESSI titration, agree within one order of magnitude with literature values. This shows that the solution phase complexes are put into the gas phase intact. However, the spray, the desolvation, and the transmission through the mass spectrometer modulate the complex to free protein ratio somewhat.

The $K_{\mathrm{D}}$ values in Table 1 increase from higher to lower charge states for ESI and ESSI. There are two reasons to explain this observation. First, the conformation of HEWL in the gas phase might be slightly different for different charge states. This has been demonstrated using ion mobility mass spectrometry on gaseous disulfide-intact lysozyme [50]. Two conformers of disulfide-intact lysozyme were found, one highly folded, the other one partially unfolded. These findings could explain the different $K_{\mathrm{D}} \mathrm{S}$ as the protein conformation and, therefore, the geometry of the binding pocket as well as the interactions with the ligand could be dependent on charge state. Second, noncovalent interaction strengths of biochemical interactions are different in solution compared with the gas phase [33]. As the dielectric constant drops when going from solution to gas phase, interactions based on charges, dipoles, and polarizability are expected to increase. In the case of HEWL binding with $\mathrm{NAG}_{3}$, there are no ion-ion interactions but only dipole interactions present in the binding pocket (hydrogen bonds between Y63, P103, W109, and NAG 3 could be shown for a HEWL mutant [51]. An increasing charge on the protein should lead to additional stabilization due to induced dipoles, causing a higher apparent affinity. This is indeed found, but there is only a significant trend for ESI, not for nanoESI or ESSI. When going from +6 to +5 for ESSI, the measured $K_{\mathrm{D}}$ increases by only $2.5 \mu \mathrm{M}$, whereas the increase for ESI when going from +8 to +7 is much higher, $26.3 \mu \mathrm{M}$. The direction of this observation is also somewhat surprising, as more expanded protein conformations with presumably lower binding affinities would be expected for more highly charged protein ions.

When comparing the same charge states for the different ionization methods, the affinity of ligand to the protein decreases from ESSI to nanoESI to ESI, respectively. The mean value reflects this trend for the three different techniques. Whereas the mean $K_{\mathrm{D}}$ determined for ESSI is $19.4 \pm 3.6 \mu \mathrm{M}$, the mean $K_{\mathrm{D}}$ for nanoESI or ESI is about a factor of two or three higher, respectively. Possibly the overall lower $K_{\mathrm{D}}$ values found

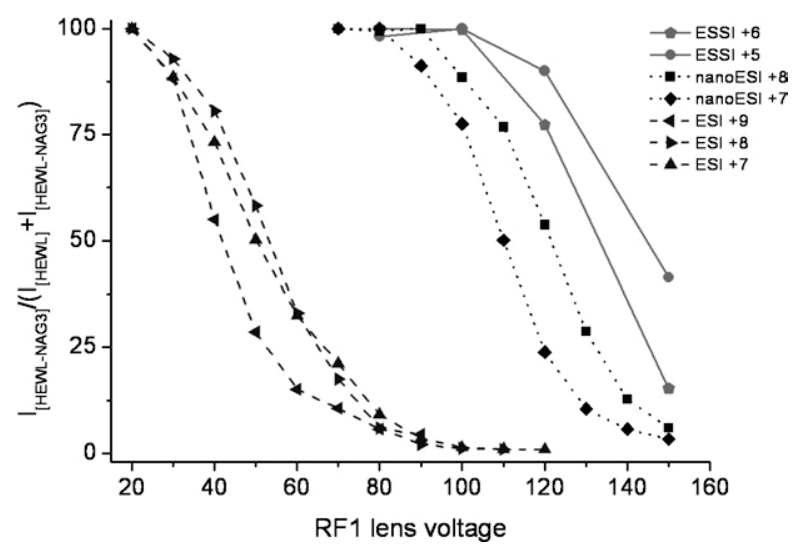

Figure 2. Percentage of HEWL-NAG3 complex over total HEWL $\left(\mathrm{I}_{\text {HEWL }}+\mathrm{I}_{\text {HEWL-NAG3 }}\right)$ is plotted against the RF1 lens voltage. The numbers in the legend indicate the charge states observed for the different ionization techniques.

for ESI are due to the higher desolvation gas temperature (as described in the Experimental section) required for the observation of noncovalent complexes.

We next investigated the influence of instrumental parameters. Figure 2 shows the influence of the RF1 voltage on the stability of the noncovalent complex between HEWL and $\mathrm{NAG}_{3}$. As the RF1 voltage is increased, the complex ions gradually dissociate. The RF1 voltage also has an influence on the charge state distribution of the noncovalent complex ions generated. The higher charge states are influenced more strongly by the RF1 parameter than the lower charge states. This is true for ESI, as the complex ions of +9 are dissociated at lower voltages compared with complex ions of +8 and +7 . It is also the case for ESSI, as the complexes with charge +6 are almost completely destroyed at a RF1 voltage of $150 \mathrm{~V}$, whereas about $50 \%$ of the complexes with charge +5 are still intact. Since the initial droplets generated by ESI are larger compared with nanoESI or ESSI, harsher instrumental conditions (higher source temperature and RF1 settings) are required to observe a well desolvated protein or complex. In this case, more energy is transferred to the large molecules, which can break the noncovalent interactions between the ligand and the protein. The compromise of sufficient desolvation versus maintenance of intact complexes is met by softer experimental conditions in the case of nanoESI and ESSI, because the initial droplet size is much smaller.

Figure 2 also shows that complex ions generated by ESI depend more on the RF1 setting compared with the other techniques, and that noncovalent complexes are completely dissociated at voltages $>40 \mathrm{~V}$. Complex ions generated by nanoESI or ESSI show a dependence on the RF1 lens only at much higher voltages. The noncovalent complex ions from nanoESI start to be destroyed at $>100 \mathrm{~V}$, whereas ESSI complex ions are affected by this parameter only at higher voltages $(>120 \mathrm{~V})$. It is important to point out that for each protein-ligand system as well as for different instrument types, opti- 


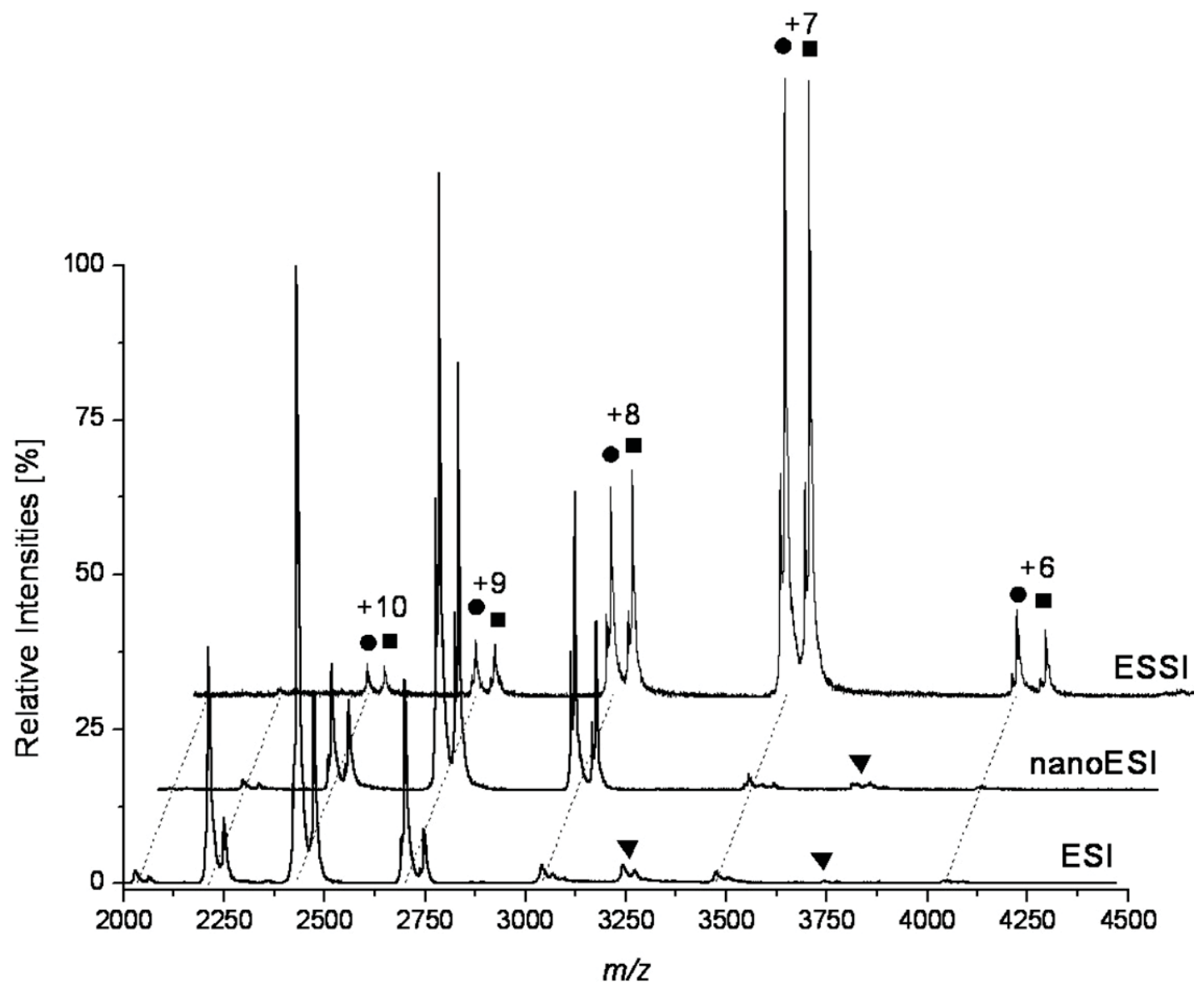

Figure 3. Representative mass spectra of the noncovalent AK-ADP complex (AK $10 \mu \mathrm{M}$, ADP $2 \mu \mathrm{M}$ in $20 \mathrm{mM}$ ammonium bicarbonate buffer) using different ionization techniques. The charge states of free protein signals (depicted as filled circle) and AK-ADP complex signals (filled square) are given for ESSI. The signals for the nonspecific homodimer of AK (inverted filled triangle) [6] were observed only for ESI and nanoESI.

mum parameters may vary. For example, the cone voltage on instruments using a linear sample infusion and ion transmission geometry seems to have a great influence on the stability of noncovalent complexes [52, 53]. Using the Z-shaped spray interface of the Q-TOF Ultima, however, we found little or no influence of the cone voltage on the stability of noncovalent complexes, neither for HEWL-NAG 3 nor for AK-ADP. These results show the importance of a careful optimization of instrumental parameters for the investigation of noncovalent interactions. The titration experiment for $\mathrm{HEWL}-\mathrm{NAG}_{3}$ described in Table 1 was repeated using ESI with an RF1 setting of $50 \mathrm{~V}$ to keep the noncovalent complexes intact. Due to incomplete desolvation and peak broadening below $50 \mathrm{~V}$, no spectra useful for titration experiment could be recorded. We determined $K_{\mathrm{D}}$ values of $12.9 \pm 0.5 \mu \mathrm{M}$ for charge state +7 and of $12.9 \pm 0.4 \mu \mathrm{M}$ for charge state +6 , which are very similar to those determined by ESSI at a RF1 voltage of $100 \mathrm{~V}$.

\section{Binding of $A K$ with $A D P$}

Representative mass spectra of the noncovalent AKADP complex obtained by ESI, nanoESI and ESSI are shown in Figure 3. These mass spectra were recorded using the same instrumental settings and sample concentrations (10 $\mu \mathrm{M}$ for $\mathrm{AK}$ and $2 \mu \mathrm{M}$ for ADP). As shown in Figure 3, the ion source affects the charge state distribution for the same protein sample. For ESI, the most abundant charge states are $+11,+10$, and +9 , with +10 being most intense. For nanoESI charge states $+10,+9$, and +8 are apparent, with +9 being the predominant one. Finally, for ESSI charge states $+9,+8$, +7 , and +6 are present and +7 is the most abundant. ESSI has previously been shown to create protein ions with a very narrow charge state distribution or even with only one predominant charge [14, 17]. However, for AK, a broader charge state distribution is observed using ESSI compared with the other ionization techniques.

Additional signals appear with low intensity at $\mathrm{m} / \mathrm{z}$ 3250 and 3750 for both ESI and nanoESI but not for ESSI (Figure 3). They were assigned to the nonspecific AK homodimer. The formation of nonspecific complexes depends on the number of analyte molecules in the progeny droplets that ultimately lead to gaseous ions. The fact that for ESSI no nonspecific homodimer was found is an indication of small initial droplets (contain- 


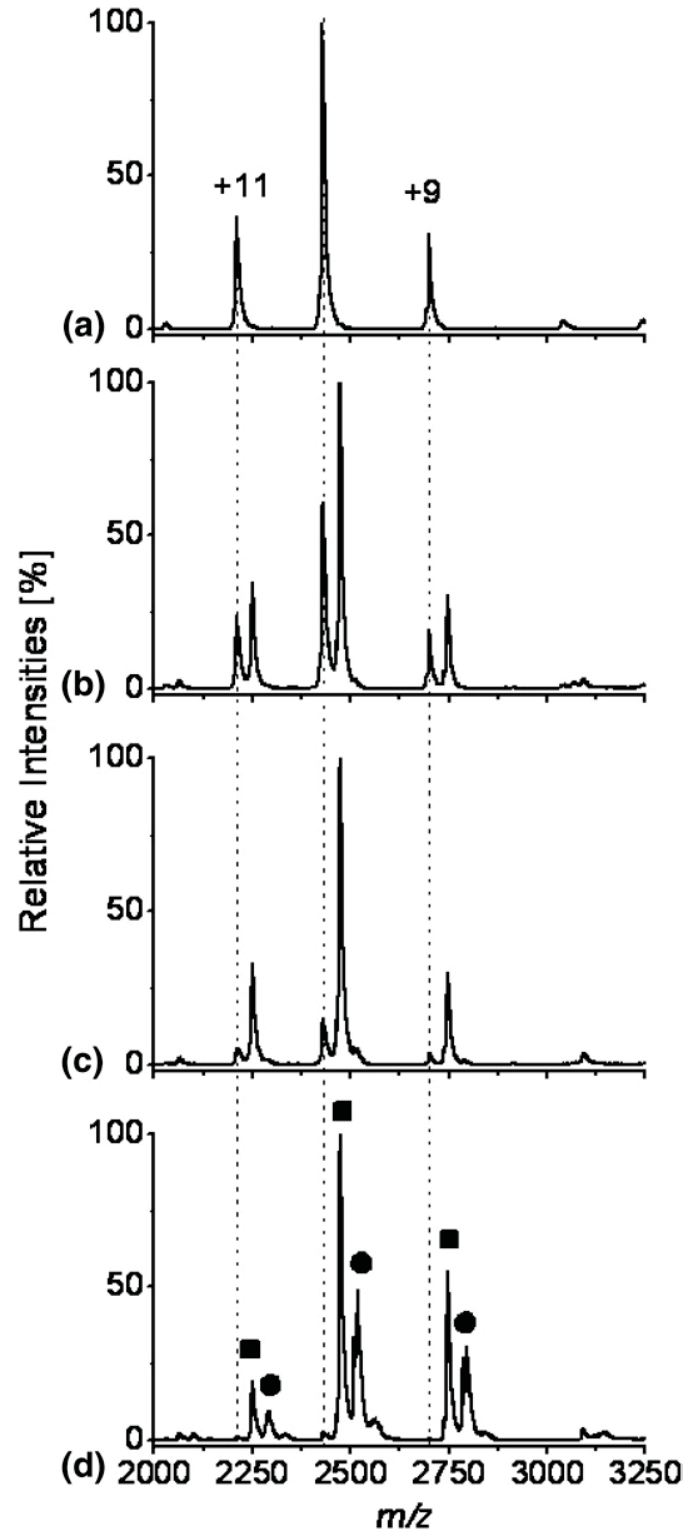

Figure 4. Representative mass spectra of AK with different ADP concentrations using nanoESI. (a) The mass spectrum of $10 \mu \mathrm{M}$ AK in absence of ADP. (b), (c), (d) The mass spectra of $10 \mu \mathrm{M}$ AK in presence of 6, 10, $50 \mu \mathrm{M}$ ADP, respectively. The lines are added to help guide the eye for the free AK, the AK-(ADP) and the $\mathrm{AK}-(\mathrm{ADP})_{2}$ species are depicted as filled square and filled circle, respectively.

ing less analyte molecules) generated due to the high shear force of the nebulizer gas. This assumption is supported by observations made when ESSI was first introduced, where it was reported to exhibit less clustering and aggregation [14].

It is known from active site-blocking experiments done earlier in this group that AK specifically binds nucleotides in ESI-MS measurements [34]. Thus, nonspecific binding can be excluded in this case. Moreover, we could not observe nonspecific clustering, even at high ADP concentrations (50 $\mu \mathrm{M}$ ADP) confirming the specificity of the complexes observed. Figure 4 shows the progress of the AK-ADP titration using nanoESI. Figures $4 b, c$, and d show the mass spectrum of AK without ADP added to the solution. Figures $4 \mathrm{~b}$ and $c$ show mass spectra of AK titrated with $6 \mu \mathrm{M}, 10 \mu \mathrm{M}$ and $50 \mu \mathrm{M}$ ADP, respectively. It is quite obvious that with $10 \mu \mathrm{M}$ ADP, full complexation of the AK is achieved, indicated by the complete mass shift to the AK-(ADP) species. If the concentration is further increased, the second binding pocket of AK also binds ADP. The peaks appear to be broader, but the integrated signals used for the $K_{\mathrm{D}}$ determination were comparable for the free $\mathrm{AK}$ and the AK-(ADP) complexes. Due to the higher $K_{\mathrm{D}}$ value even at high ADP concentrations some AK-(ADP) will remain uncomplexed.

Figure 5 shows the fitted titration curves for the AK-ADP experiments using different ionization techniques. Figure 5 a shows the titration of the first binding site of AK for charge state +9 . The same trend was observed by comparing charge state +10 for the different ionization methods (data not shown). The intensity ratio of free protein over complex is plotted against the initial ligand concentration. At concentrations higher than $10 \mu \mathrm{M}$, the ratio of free protein over complex remains constant (for all three ionization methods) indicating that the protein species in solution is completely saturated with ligand (Figure 5a). If the ligand concentration is further increased $(>10 \mu \mathrm{M})$, an additional signal appears in the mass spectrum corresponding to the AK-(ADP) $)_{2}$ complex. Figure $5 \mathrm{~b}$ shows the titration of the second binding pocket of $\mathrm{AK}$ by monitoring the ratio of AK-(ADP) over AK-(ADP) $)_{2}$ against the ligand concentration. In both cases (Figures $5 \mathrm{a}$ and b) ESSI show the highest affinity, followed by nanoESI and then ESI. This can again be attributed to the fast and more complete desolvation process of ESSI ions and the better conservation of the noncovalent complexes during their path from solution into the gas phase. The droplet evolution to from ions produced by ESI is considerably different compared with nanoESI and ESSI $[8,2$, 14]. Starting at much bigger initial droplets, the desolvation process in the case of ESI is likely much slower and the number of Coulombic fissions required is much higher. This leads to only partial desolvation in the atmospheric pressure region and often further desolvation and ion formation happens in the first part of the mass spectrometer. The fact that a high source temperature had to be set for ESI (see the Experimental section) compared with the other two techniques supports this interpretation.

The $K_{\mathrm{D}}$ values obtained for the first and second binding pocket of AK binding ADP in the absence of magnesium are shown in Table $2 a$ and $b$, respectively. The $K_{\mathrm{D}}$ values for the first active site are all in the range of 1.8 to $5.7 \mu \mathrm{M}$ for the ionization techniques investigated. By comparing the $K_{\mathrm{D}} \mathrm{s}$ for charge state +9 for all ionization methods, ESSI has the lowest values compared with the others. In fact, the $K_{\mathrm{D}} \mathrm{S}$ obtained for ESI are more than a factor of two higher than for ESSI. Again, the higher $K_{\mathrm{D}}$ found for ESI may be due to the 

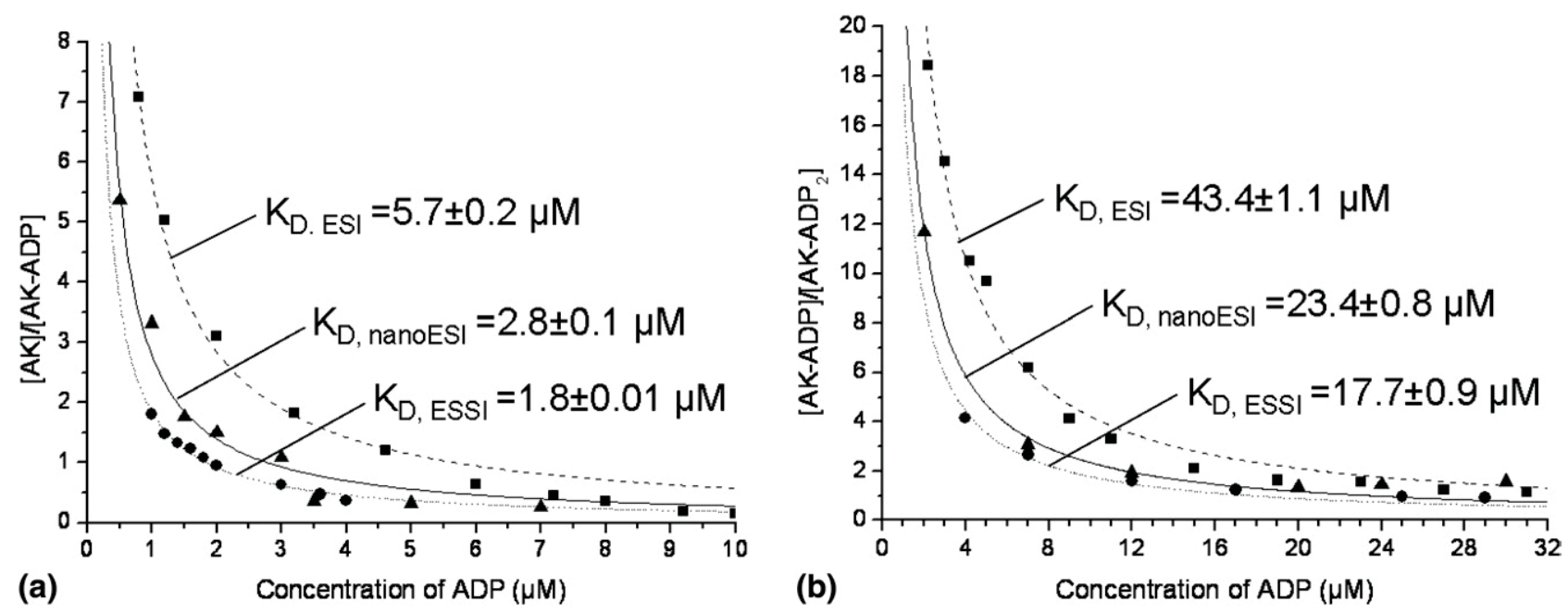

Figure 5. Titration curves for AK with ADP of charge state 9+ using different ionization techniques. ESI is depicted as filled square, nanoESI as filled triangle, and ESSI as filled circle. (a) The titration of AK with ADP of the first binding site. (b) The titration of the second binding site.

required elevated desolvation gas temperature, as explained in the Experimental section. The same phenomenon is observed by comparing charge state +10 and +9 in Table $2 b$, where this difference is even larger than a factor of two. To our knowledge, no data has been published on $K_{\mathrm{D}}$ values for the binding of ADP to AK, even though several crystal structures have been determined for the noncovalent complex between $\mathrm{AK}$ and ADP [54, 55]. There are two different, independent binding sites on AK [44]; one binding site for ATP and one for AMP. Both site, however, are also capable of binding ADP, which has been reported for Mycobacterium tuberculosis AK [54]. Moreover, large domain movements and substrate-induced conformational changes upon substrate binding have been reported, implying that there are cooperative effects present when occupying the second binding site [44]. In our work, we can observe quite clearly that the second binding pocket is "activated" upon ADP binding of the first binding site. This activated AK-ADP complex is then capable of binding ADP in the second binding site with an affinity about one order of magnitude lower compared with the first ADP bound.

In Table 2, the same general tendency was observed regarding the charge state dependence of the $K_{\mathrm{D}}$ values, as in the case of the HEWL-NAG ${ }_{3}$ titration (see Table 2). This trend was found for all three ionization methods examined. The $K_{\mathrm{D}}$ values increase with decreasing charge state (see Table 2a), a trend even more apparent for the titration of the second binding pocket (Table $2 b$ ) where the difference can be greater than $7 \mu \mathrm{M}$ in $K_{\mathrm{D}}$ going from charge state +10 to +6 for ESSI. Regarding this general tendency, there are again differences for the different ionization methods. Whereas the change in $K_{\mathrm{D}}$ going from +10 to +8 is between $0.2 \mu \mathrm{M}$ (Table $2 \mathrm{a}$ ) and $4.7 \mu \mathrm{M}$ (Table $2 \mathrm{~b}$ ) for ESSI, the change for ESI by going from +11 to +9 is considerably greater, $1.9 \mu \mathrm{M}$ (Table 2a) and $6.8 \mu \mathrm{M}$ (Table $2 \mathrm{~b}$ ). In other words, the charge state dependent $K_{\mathrm{D}}$ values obtained for ESSI are more consistent within a broad range of charge states compared with, e.g., ESI. In this case, ionic interactions between the phosphates of ADP and the AK binding

Table 2. Calculated dissociation constants $\left(\mathrm{K}_{\mathrm{D}} \mathrm{s}\right)$ in $\mu \mathrm{M}$ for different charge states from the titration experiments of AK with ADP. (a) shows the obtained $K_{D} s$ for the first binding site of $A K,(b)$ shows the determined $K_{D} s$ for the second binding site. The errors show the fitting quality of the titration curve

\begin{tabular}{|c|c|c|c|c|c|c|c|}
\hline $\begin{array}{c}\text { a } \\
\text { Charge state }\end{array}$ & +11 & +10 & +9 & +8 & +7 & +6 & Mean $K_{D}$ \\
\hline ESSI & & $1.9 \pm 0.1$ & $1.8 \pm 0.01$ & $2.1 \pm 0.1$ & $2.3 \pm 0.1$ & $2.7 \pm 0.1$ & $2.2 \pm 0.8$ \\
\hline NanoESI & & $2.0 \pm 0.2$ & $2.8 \pm 0.1$ & $3.6 \pm 0.2$ & & & $2.8 \pm 1.0$ \\
\hline ESI & $3.8 \pm 0.2$ & $4.7 \pm 0.1$ & $5.7 \pm 0.2$ & & & & $4.7 \pm 1.0$ \\
\hline \multicolumn{8}{|l|}{ b } \\
\hline Charge state & +11 & +10 & +9 & +8 & +7 & +6 & Mean $\mathrm{K}_{\mathrm{D}}$ \\
\hline ESSI & & $15.0 \pm 0.9$ & $17.7 \pm 0.9$ & $19.7 \pm 0.8$ & $23.1 \pm 0.6$ & $22.1 \pm 0.8$ & $19.5 \pm 8.0$ \\
\hline NanoESI & & & $23.4 \pm 0.8$ & $23.0 \pm 0.8$ & & & $23.2 \pm 3.2$ \\
\hline $\mathrm{ESI}$ & $36.6 \pm 1.8$ & $40.8 \pm 1.4$ & $43.4 \pm 1.1$ & & & & $40.3 \pm 5.0$ \\
\hline
\end{tabular}


pockets are present $[54,55]$. As reported in the crystal structures, in the AK binding pocket (depending on the organism) there are different arginines and lysines present, which stabilize the phosphates of the nucleotides. This fact can again be discussed in terms of a modulation of the relative intensities of complex and free protein signal due to different interaction strengths in the gas phase compared with solution. The trend of increasing ligand affinity with increasing charge on the protein can be explained by stronger electrostatic forces between a more highly charged protein ion interacting with a ligand carrying an opposite charge [33]. Nevertheless, as in the case of HEWL-NAG ${ }_{3}$, this change in interaction strength must be rather weak, since the variation in $K_{\mathrm{D}}$ with charge state is small. It can also be questioned whether the ionization efficiency of protein ions is constant when ADP is bound. Ionization efficiency and ion formation in ESI is partially governed by protonation of basic residues (arginines, lysines, and histidines) of the proteins [56,57]. When ADP is bound in the binding pocket, however, the arginines and lysines present would be shielded by the ligand and their protonation would be prevented. If, however, these residues would take part in the protonation of the protein during the electrospray process, a shift in average charge state would be expected when ligand is added, as less protonation sites are available. No such a shift was observed in our experiments, which led us to conclude that the basic residues in the binding pocket are not involved in the protonation of the protein during the electrospray process.

\section{Binding of LCK with Compound A}

In terms of sample consumption, ESI and ESSI are comparable: for one 3-min measurement and a flow rate of $5 \mu \mathrm{L} / \mathrm{min}$, both techniques require a sample volume of 15 to $20 \mu \mathrm{L}$. However, to have mass spectra of comparable intensity with nanoESI, a sample volume of only 2 to $3 \mu \mathrm{L}$ is needed. Comparing the spray stability of the ionization techniques used, ESSI has by far the most stable spray over time, followed by nanoESI. It is quite difficult to achieve a stable spray mode with ESI using aqueous solutions, and the required voltage window to accomplish this is quite narrow [3, 4]. The advantage of nanoESI using the NanoMate is clearly its ease of use and the exceptionally low sample consumption, which makes it even more attractive when dealing with expensive and precious samples. This was the case for our LCK sample and, therefore, we chose to perform the titration experiments using only the NanoMate to determine this protein's binding constant to Compound A.

Figure 6 shows four representative mass spectra of LCK under nondenaturing conditions with various amounts of Compound A. Figure 6a shows the mass spectrum obtained for $5 \mu \mathrm{M}$ LCK in absence of ligand: charge states $+11,+10$, and +9 were observed. Figures $6 \mathrm{~b}-\mathrm{d}$ show mass spectra obtained for the noncovalent

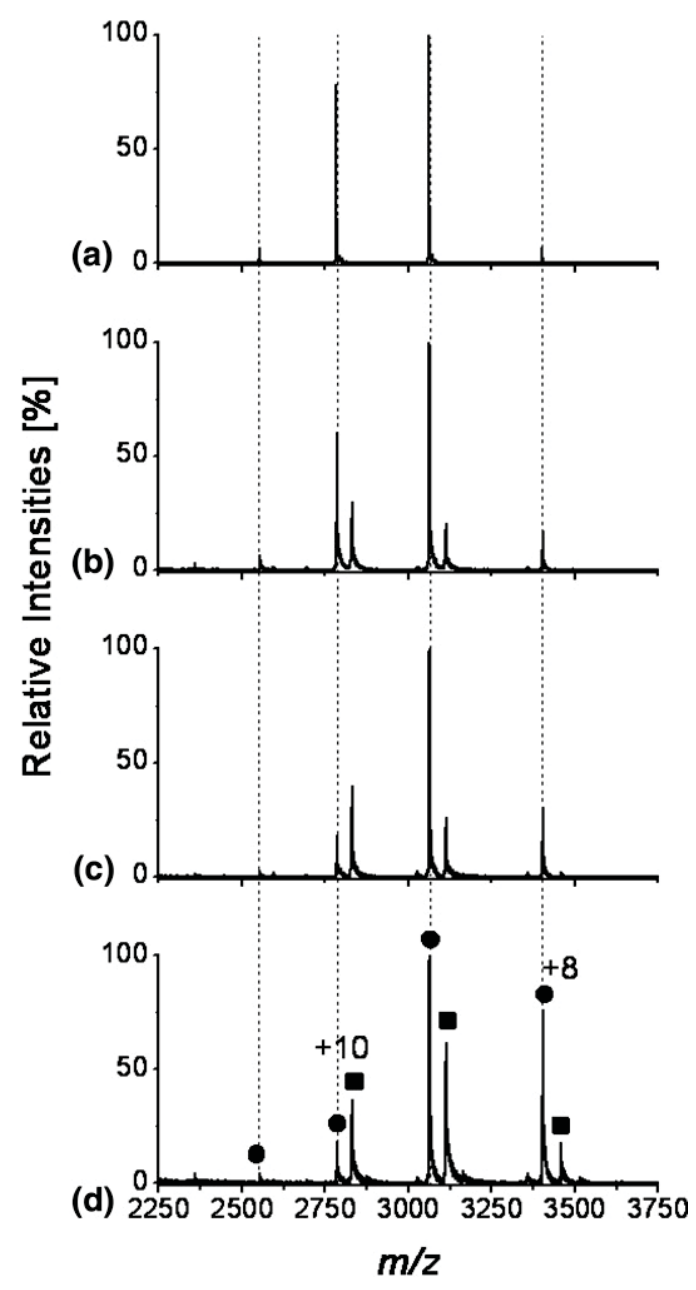

Figure 6. Representative mass spectra using nanoESI of the LCK-Compound A titration experiment (LCK $5 \mu \mathrm{M}$, in $20 \mathrm{mM}$ ammonium bicarbonate buffer at $\mathrm{pH}$ 6.8). (a) shows the mass spectrum obtained of LCK sprayed under nondenaturing conditions in absence of Compound A. Mass spectra of the titration points with 1, 10, and $50 \mu \mathrm{M}$ Compound A are shown in (b), (c), and (d), respectively. In (d), charge states and corresponding free protein signals (filled circle) and noncovalent LCK-Compound A complex (filled square) are depicted.

LCK-Compound A complex, for three different concentrations. In this case the complex abundance is very different for the different charge states. For charge state +10 the ratio of free protein to complex is around 2:1, it decreases going to charge state +9 and for +8 no complex signal appears at this concentration.

Another interesting aspect is that the charge state distribution is slightly shifted by adding ligand, i.e., charge state +8 is not apparent for LCK in the absence of Compound A, but by adding $10 \mu \mathrm{M}$ Compound $\mathrm{A}$ to the sample (Figure $6 \mathrm{c}$ ) charge state +11 disappears and +8 becomes more dominant compared with Figure $6 \mathrm{~b}$. Again, the ratio of free protein to complex is strongly charge state dependent, as for +10 the ratio now is around 1:2 whereas for +9 the ratio does not change compared with Figure $6 \mathrm{~b}$, and for +8 still no complex signal is observed. With increasing Compound A con- 
centration ( $50 \mu \mathrm{M}$, Figure $6 \mathrm{~d}$ ) this ratio appears to be constant for +10 , it decreased for +9 , and finally for +8 a complex signal emerged. With charge state +9 being the most intense and +8 at about $80 \%$ relative intensity, the overall charge state distribution is shifted a lot compared with Figure 6a.

We performed a titration experiment using concentrations of Compound A ranging up to $500 \mu \mathrm{M}$, which was necessary to get useful ratios for free protein to complex for charge state +9 . We determined the $K_{\mathrm{D}}$ value for each charge state using the method described in the Experimental section. The calculated $K_{\mathrm{D}} \mathrm{S}$ were $142 \pm 9 \mu \mathrm{M}$ for charge state $+9,15.3 \pm 0.8 \mu \mathrm{M}$ for +10 , and $1.12 \pm 0.06 \mu \mathrm{M}$ for +11 . The trend to lower affinity with decreasing charge on the protein is very strong in this case, about one order of magnitude between subsequent charge states. Finally, there is a large discrepancy between our measurements and known $K_{\mathrm{D}}$ values (nM range) obtained by measurements in solution.

Overall, this strong charge state dependence indicates that the complex is not well behaved in these experiments; ESI titration experiments are not well suited for assessing $K_{\mathrm{D}}$ values in such a case. Possible explanations for this behavior could be: (1) As discussed for lysozyme, there could be more than one LCK conformation in the gas phase, depending on the charge state. If the binding pocket conformation is changed a lot with increasing charge on the protein, different complex abundances comparing charge states could result. Unfortunately, no ion mobility data are available for LCK up to now. (2) A completely different interaction may exist in the gas phase compared with solution behavior. By looking at structures of complexes with other ligands competing for the ATP-binding site of LCK, the residues that take part in the ligand binding can be identified. Zhu et al. [45] have shown that for the competitive ligands AMP-PNP (a nonselective, nonhydrolyzable ATP analog), staurosporine (a potent but nonselective protein kinase inhibitor), and PP2 (a potent SRC-family selective protein tyrosine kinase inhibitor), hydrogen bond interactions (E317, M319, S323, and T316, E317, M319, respectively), and several dispersion forces can be attributed to the residues in the binding pocket. K273, which is part of the binding pocket, is assumed to play a key role for the phenomena in the gas phase. (3) Binding of Compound A can prevent protonation of basic residues in (or near) the binding pocket (e.g., K273), thus affecting the charge state and ionization efficiency of ligand-bound protein. This interpretation is consistent with the overall shift to lower charge with increasing ligand concentration (Figure 6). The large change in the $K_{\mathrm{D}}$ values derived from different charge states, and the overall shift in average charge state with increasing amount of ligand could perhaps be dealt with by using a response factor accounting for the different ionization efficiencies. As shown by Gabelica et al. [38], one titration curve can be fitted to give $K_{\mathrm{D}} \mathrm{S}$ and response factors orders of magnitudes off but still at very good fitting quality. However, in the present case, different response factors for each charge state would have to be used, leading to several additional fitting parameters for the $K_{\mathrm{D}}$ calculation, a procedure expected to be quite prone to error.

\section{Conclusions}

We compared the performance of ESI, nanoESI, and ESSI for $\mathrm{K}_{\mathrm{D}}$ determination with mass spectrometry. For the model system HEWL binding $\mathrm{NAG}_{3}$, we found that all three ionization techniques investigated here gave $\mathrm{K}_{\mathrm{D}}$ values that were similar and in good agreement with the literature values observed in solution phase. ESSI, however, yielded the best $K_{\mathrm{D}}$ values compared with solution data, which is evidence for the softness of this method [14, 17]. The study of the influence of instrumental parameters on the complex stability revealed that ESSI ions are significantly less prone to gas-phase dissociation compared with the other ion sources.

We then determined the binding affinity of ADP binding to the two nucleotide binding pockets of $\mathrm{AK}$. The ionization methods examined gave very similar $K_{\mathrm{D}}$ values, again with ESSI providing the lowest values, 2.2 $\pm 0.8 \mu \mathrm{M}$ for the first and $19.5 \pm 8.0 \mu \mathrm{M}$ for the second binding site. We conclude that the droplet evaporation process is considerably different for ESSI compared with nanoESI and conventional ESI. For ESI, there is a droplet shrinking process accompanied by Coulomb droplet fissions and evaporation, eventually leading to fully desolvated ions [56,57]. A very similar process is proposed for nanoESI, assuming that fewer fissions occur that the evaporation is faster, because nanoESI starts from smaller initial droplets [8]. For ESSI however, the ion formation from charged droplets seems to be substantially different as the whole process is driven by the sonic sheath gas. The time scale of the evaporation in ESSI from the analyte in a charged droplet to the fully desolvated ion is expected be a lot shorter compared with the other spray methods. This feature together, with the low internal energy of the ions, might be the reason for the unique spectral characteristics obtained from ESSI experiments.

For both systems, HEWL-NAG 3 and AK-ADP, we found a weak charge state dependence of the determined $K_{\mathrm{D}}$ values-an increasing affinity with increasing charge on the protein. Nonspecific interactions due to electrostatic effects the gas phase, however, could be excluded for the HEWL and AK systems investigated here.

Our experiments with LCK binding Compound A demonstrate that a reliable $K_{\mathrm{D}}$ determination is difficult when dealing with a large charge state dependence of complex abundance. The following criteria can be used to judge whether one should expect a $K_{\mathrm{D}}$ determination to be difficult or impossible: (1) when a ligand significantly alters the ionization efficiency of its protein target, and a shift in average charge state distribution results, and (2) if the $K_{\mathrm{D}}$ values determined for individ- 
ual charge states increase a lot (by one order of magnitude) between adjacent charge states. In such a case, the introduction of a response factor can be reasonable. However, the introduction of charge state dependent response factors provides several additional fitting parameters, which would leave calculated $K_{\mathrm{D}} \mathrm{s}$ even more questionable.

\section{Acknowledgments}

MCJ and DT thank Novartis Institutes for BioMedical Research for financial support and sample donation. MCJ thanks Kurt Baumgartner from the ETH machine shop for his assistance in setting up the electrosonic spray source.

\section{References}

1. Fenn, J. B.; Mann, M.; Meng, C. K.; Wong, S. F.; Whitehouse, C. M. Electrospray Ionization for Mass-Spectrometry of Large Biomolecules. Science 1989, 246, 64-71.

2. Gross, J. H. Mass Spectrometry. A Textbook, 2nd ed.; Springer-Verlag: Berlin and Heidelberg, 2004; 441-474.

3. Cloupeau, M.; Prunetfoch, B. Electrohydrodynamic Spraying Functioning Modes-A Critical-Review. J. Aerosol Sci. 1994, 25, 1021-1036.

4. Parvin, L.; Galicia, M. C.; Gauntt, J. M.; Carney, L. M.; Nguyen, A. B. Park, E.; Heffernan, L.; Vertes, A. Electrospray Diagnostics by Fourier Analysis of Current Oscillations and Fast Imaging. Anal. Chem. 2005, 77, 3908-3915.

5. Nemes, P.; Marginean, I.; Vertes, A. Spraying Mode Effect on Droplet Formation and Ion Chemistry in Electrosprays. Anal. Chem. 2007, 79, 3105-3116.

6. Bruins, A. P.; Covey, T. R.; Henion, J. D. Ion Spray Interface for Combined Liquid Chromatography/Atmospheric Pressure Ionization Mass-Spectrometry. Anal. Chem. 1987, 59, 2642-2646.

7. Wilm, M. S.; Mann, M. Electrospray and Taylor-Cone Theory, Doles Beam of Macromolecules at Last. Int. J. Mass Spectrom Ion Processes 1994, 136, 167-180.

8. Wilm, M.; Mann, M. Analytical Properties of the Nanoelectrospray Ion Source. Anal. Chem. 1996, 68, 1-8.

9. Wilm, M.; Shevchenko, A.; Houthaeve, T.; Breit, S.; Schweigerer, L.; Fotsis, T.; Mann, M. Femtomole Sequencing of Proteins from Polyacrylamide Gels by Nano-Electrospray Mass Spectrometry. Nature 1996, 379, $466-469$.

10. Schultz, G. A.; Corso, T. N.; Prosser, S. J.; Zhang, S. A Fully Integrated Monolithic Microchip Electrospray Device for Mass Spectrometry. Anal. Chem. 2000, 72, 4058-4063.

11. Zhang, S.; Van Pelt, C. K. Chip-Based Nanoelectrospray Mass Spectrometry for Protein Characterization. Expert Rev. Proteom. 2004, 1, $449-468$.

12. Kebarle, P.; Tang, L. From Ions in Solution to Ions in the Gas Phase-the Mechanism of Electrospray Mass-Spectrometry. Anal. Chem. 1993, 65, A972-A986.

13. Juraschek, R.; Dulcks, T.; Karas, M. Nanoelectrospray- More than Just a Minimized-Flow Electrospray Ionization Source. J. Am Soc. Mass Spectrom. 1999, 10, 300-308.

14. Takats, Z.; Wiseman, J. M.; Gologan, B.; Cooks, R. G. Electrosonic Spray Ionization. A Gentle Technique for Generating Folded Proteins and Protein Complexes in the Gas Phase and for Studying Ion-Molecule Reactions at Atmospheric Pressure. Anal. Chem. 2004, 76, 4050-4058.

15. Hirabayashi, A.; Sakairi, M.; Koizumi, H. Sonic Spray Ionization Method for Atmospheric-Pressure Ionization Mass-Spectrometry. Anal. Chem. 1994, 66, 4557-4559.

16. Hirabayashi, A.; Sakairi, M.; Koizumi, H. Sonic Spray Mass-Spectrometry. Anal. Chem. 1995, 67, 2878-2882.

17. Wiseman, J. M.; Takats, Z.; Gologan, B.; Davisson, V. J.; Cooks, R. G. Direct Characterization of Enzyme-Substrate Complexes by Using Electrosonic Spray Ionization Mass Spectrometry. Angew. Chem. Int. Ed. 2005, 44, 913-916.

18. Ganem, B.; Li, Y. T.; Henion, J. D. Observation of Noncovalent Enzyme Substrate and Enzyme Product Complexes by Ion-Spray Mass-Spectrometry. J. Am. Chem. Soc. 1991, 113, 7818-7819.

19. Smith, R. D.; Lightwahl, K. J. The Observation of Noncovalent Interactions in Solution by Electrospray-Ionization Mass-SpectrometryPromise, Pitfalls, and Prognosis. Biol. Mass Spectrom. 1993, 22, 493-501.

20. Loo, J. A. Studying Noncovalent Protein Complexes by Electrospray Ionization Mass Spectrometry. Mass Spectrom. Rev. 1997, 16, 1-23.

21. Schnier, P. D.; Klassen, J. S.; Strittmatter, E. E.; Williams, E. R. Activation Energies for Dissociation of Double Strand Oligonucleotide Anions: Evidence for Watson-Crick Base Pairing in Vacuo. J. Am. Chem. Soc. 1998, 120, 9605-9613.
22. Heck, A. J. R.; van den Heuvel, R. H. H. Investigation of Intact Protein Complexes by Mass Spectrometry. Mass Spectrom. Rev. 2004, 23, $368-$ 389.

23. Ashcroft, A. E. Recent Developments in Electrospray Ionization Mass Spectrometry: Noncovalently Bound Protein Complexes. Nat. Prod. Rep. 2005, 22, 452-464.

24. Hofstadler, S. A.; Sannes-Lowery, K. A. Applications of ESI-MS in Drug Discovery: Interrogation of Noncovalent Complexes. Nat. Rev. Drug Discov. 2006, 5, 585-595.

25. Cunniff, J. B.; Vouros, P. False Positives and the Detection of Cyclodextrin Inclusion Complexes by Electrospray Mass-Spectrometry. J. Am. Soc. Mass Spectrom. 1995, 6, 437-447.

26. Kitova, E. N.; Wang, W. J.; Bundle, D. R.; Klassen, J. S. Retention of Bioactive Ligand Conformation in a Gaseous Protein-Trisaccharide Complex. J. Am. Chem. Soc. 2002, 124, 13980-13981.

27. Hossain, B. M.; Simmons, D. A.; Konermann, L. Do Electrospray Mass Spectra Reflect the Ligand Binding State of Proteins in Solution? Can. J. Chem. Rev. 2005, 83, 1953-1960.

28. Iavarone, A. T.; Parks, J. H. Conformational Change in Unsolvated Trp-Cage Protein Probed by Fluorescence. J. Am. Chem. Soc. 2005, 127, 8606-8607.

29. Ruotolo, B. T.; Giles, K.; Campuzano, I.; Sandercock, A. M.; Bateman, R. H.; Robinson, C. V. Evidence for Macromolecular Protein Rings in the Absence of Bulk Water. Science 2005, 310, 1658-1661.

30. Benesch, J. L.; Robinson, C. V. Mass spectrometry of Macromolecular Assemblies: Preservation and Dissociation. Curr. Opin. Struct. Biol. 2006, 16, 245-251.

31. Ruotolo, B. T.; Robinson, C. V. Aspects of Native Proteins are Retained in Vacuum. Curr. Opin. Chem. Biol. 2006, 10, 402-408.

32. Wortmann, A.; Kistler-Momotova, A.; Zenobi, R.; Heine, M. C.; Wilhelm, O.; Pratsinis, S. E. Shrinking Droplets in Electrospray Ionization and Their Influence on Chemical Equilibria. J. Am. Soc. Mass Spectrom. 2007, 18, 385-393.

33. Daniel, J. M.; Friess, S. D.; Rajagopalan, S.; Wendt, S.; Zenobi, R. Quantitative Determination of Noncovalent Binding Interactions Using Soft Ionization Mass Spectrometry. Int. J. Mass Spectrom. 2002, 216, 1-27.

34. Daniel, J. M.; McCombie, G.; Wendt, S.; Zenobi, R. Mass Spectrometric Determination of Association Constants of Adenylate Kinase with Two Noncovalent Inhibitors. J. Am. Soc. Mass Spectrom. 2003, 14, 442-448.

35. Wendt, S.; McCombie, G.; Daniel, J.; Kienhofer, A.; Hilvert, D.; Zenobi, R. Quantitative Evaluation of Noncovalent Chorismate Mutase-Inhibitor Binding by ESI-MS. J. Am. Soc. Mass Spectrom. 2003, 14, 1470-1476.

36. Tjernberg, A.; Carno, S.; Oliv, F.; Benkestock, K.; Edlund, P. O.; Griffiths, W. J.; Hallen, D. Determination of Dissociation Constants for ProteinLigand Complexes by Electrospray Ionization Mass Spectrometry. Anal. Chem. 2004, 76, 4325-4331.

37. Wortmann, A.; Rossi, F.; Lelais, G.; Zenobi, R. Determination of Zinc to $\beta$-Peptide Binding Constants with Electrospray Ionization Mass Spectrometry. J. Mass Spectrom. 2005, 40, 777-784.

38. Gabelica, V.; Galic, N.; Rosu, F.; Houssier, C.; De Pauw, E. Influence of Response Factors on Determining Equilibrium Association Constants of Noncovalent Complexes by Electrospray Ionization Mass Spectrometry. J. Mass Spectrom. 2003, 38, 491-501.

39. Chitta, R. K.; Rempel, D. L.; Gross, M. L. Determination of Affinity Constants and Response Factors of the Noncovalent Dimer of Gramicidin by Electrospray Ionization Mass Spectrometry and Mathematical Modeling. J. Am. Soc. Mass Spectrom. 2005, 16, 1031-1038.

40. Daubenfeld, T.; Bouin, A. P.; van der Rest, G. A deconvolution method for the separation of specific versus nonspecific interactions in noncovalent protein-ligand complexes analyzed by ESI-FT-ICR mass spectrometry. J. Am. Soc. Mass Spectrom. 2006, 17, 1239-1248.

41. Sun, J. X.; Kitova, E. N.; Wang, W. J.; Klassen, J. S. Method for Distinguishing Specific from Nonspecific Protein-Ligand Complexes in Nanoelectrospray Ionization Mass Spectrometry. Anal. Chem. 2006, 78, 3010-3018.

42. Fukamizo, T. Chitinolytic Enzymes: Catalysis, Substrate Binding, and Their Application. Curr. Protein Pept. Sci. 2000, 1, 105-124.

43. Dennhart, N.; Letzel, T. Mass Spectrometric Real-Time Monitoring of Enzymatic Glycosidic Hydrolysis, Enzymatic Inhibition, and Enzyme Complexes. Anal. Bioanal. Chem. 2006, 386, 689-698.

44. Yan, H.; Tsai, M.-D. Mechanism of Enzyme Action, Part A, Vol. 73; John Wiley and Sons: Gainesville, FL, 1999, pp. 103-134.

45. Zhu, X. T.; Kim, J. L.; Newcomb, J. R.; Rose, P. E.; Stover, D. R.; Toledo, L. M.; Zhao, H. L.; Morgenstern, K. A. Structural Analysis of the Lymphocyte-Specific Kinase Lck in Complex with Nonselective and Src Family Selective Kinase Inhibitors. Struct. Folding Design 1999, 7, 651661.

46. Fabian, M. A.; Biggs, W. H.; Treiber, D. K.; Atteridge, C. E.; Azimioara, M. D.; Benedetti, M. G.; Carter, T. A.; Ciceri, P.; Edeen, P. T.; Floyd, M. Ford, J. M.; Galvin, M.; Gerlach, J. L.; Grotzfeld, R. M.; Herrgard, S.; Insko, D. E.; Insko, M. A.; Lai, A. G.; Lelias, J. M.; Mehta, S. A.; Milanov, Z. V.; Velasco, A. M.; Wodicka, L. M.; Patel, H. K.; Zarrinkar, P. P.; Lockhart, D. J. A Small Molecule-Kinase Interaction Map for Clinical Kinase Inhibitors. Nat. Biotechnol. 2005, 23, 329-336.

47. Clark, S. M.; Konermann, L. Determination of Ligand-Protein Dissociation Constants by electrospray Mass Spectrometry-Based Diffusion Measurements. Anal. Chem. 2004, 76, 7077-7083.

48. Imoto, T.; Johnson, L. N.; North, A. C. T.; Phillips, D. C.; Rupley, J. A Vertebrate Lysozymes, 3rd ed.; Academic: New York, 1972; 665-868. 
49. Schindler, M.; Assaf, Y.; Sharon, N.; Chipman, D. M. Mechanism of Lysozyme Catalysis-Role of Ground-State Strain in Subsite-D in Hen Egg-White and Human Lysozymes. Biochemistry 1977, 16, 423-431.

50. Valentine, S. J.; Anderson, J. G.; Ellington, A. D.; Clemmer, D. E. DisulfideIntact and -Reduced Lysozyme in the Gas Phase: Conformations and Pathways of Folding and Unfolding. J. Phys. Chem. B 1997, 101, 3891-3900.

51. RCSB Protein data bank, e. B.

52. Hunter, C. L.; Mauk, A. G.; Douglas, D. J. Dissociation of Heme from Myoglobin and Cytochrome $b(5)$ : Comparison of Behavior in Solution and the Gas Phase. Biochemistry 1997, 36, 1018-1025.

53. Rogniaux, H.; Van Dorsselaer, A.; Barth, P.; Biellmann, J. F.; Barbanton, J.; van Zandt, M.; Chevrier, B.; Howard, E.; Mitschler, A.; Potier, N.; Urzhumtseva, L.; Moras, D.; Podjarny, A. Binding of Aldose Reductase Inhibitors: Correlation of Crystallographic and Mass Spectrometric Studies. J. Am. Soc. Mass Spectrom. 1999, 10, 635-647.
54. Bellinzoni, M.; Haouz, A.; Grana, M.; Munier-Lehmann, H.; Shepard, W.; Alzari, P. M. The Crystal Structure of Mycobacterium tuberculosis Adenylate Kinase in Complex with Two Molecules of ADP and Mg2+ Supports an Associative Mechanism for Phosphoryl Transfer. Protein Sci. 2006, 15, 1489-1493.

55. Berry, M. B.; Bae, E. Y.; Bilderback, T. R.; Glaser, M.; Phillips, G. N. Crystal Structure of ADP/AMP Complex of Escherichia coli Adenylate Kinase. Proteins Struct. Funct. Bioinformatics 2006, 62, 555-556.

56. Kebarle, P. A Brief Overview of the Present Status of the Mechanisms Involved in Electrospray Mass Spectrometry. J. Mass Spectrom. 2000, 35 804-817.

57. Peschke, M.; Verkerk, U. H.; Kebarle, P. Features of the ESI Mechanism that Affect the Observation of Multiply Charged Noncovalent Protein Complexes and the Determination of the Association Constant by the Titration Method. J. Am. Soc. Mass Spectrom. 2004, 15, 1424-1434. 